\title{
Epidemiological Characteristics and Genetic Diversity of Chicken Infectious Anemia Virus (CIAV) in Guangdong Province, China
}

\section{Fancong Zeng}

Foshan University School of Life Science

\section{Wenfeng Li}

Foshan University School of Life Science

Junhong Ke

Foshan University School of Life Science

\section{Feng Wen}

Foshan University School of Life Science

Jinyue Guo

Foshan University School of Life Science

\section{Xuelian Zhang}

Foshan University School of Life Science

Shujiang Huang ( $\nabla$ huangshujian@fosu.edu.cn )

Foshan University School of Life Science

\section{Kun Mei}

Foshan University School of Life Science https://orcid.org/0000-0002-2693-9185

\section{Research Article}

Keywords: CIAV, Serological survey, MSB1, Phylogenetic analysis

Posted Date: April 7th, 2021

DOI: https://doi.org/10.21203/rs.3.rs-385481/v1

License: (1) (1) This work is licensed under a Creative Commons Attribution 4.0 International License. Read Full License 


\section{Abstract}

Chicken infectious anemia virus (CIAV) is an immunosuppressive disease that causes enormous economic loss to the poultry industry worldwide, transmitted vertically and horizontally. In recent years, CIAV outbreaks have frequently occurred in southern China since 2014, especially in the Guangdong province. However, there is a lack of epidemiological studies of CIAV in Guangdong province. We performed PCR by using the serum and tissue samples collected from seven cities of Guangdong province. The PCR-positive tissue samples were inoculated into SPF eggs and MSB1 cells for virus isolation by blind-passages three times. This study isolated and characterized 325 serum samples and four tissue samples, the total positive CIAV rate of $62.15 \%$ in serum samples, and all four tissues were positive for the CIAV. However, the virus could only be isolated from MSB1 cells. Sequence analysis revealed that the four isolated comparisons with those in the GenBank showed high nucleotide similarities and were near clade $\mathrm{C} 2$. Our data showed over $60 \%$ of positive cases for CIAV serological analysis consist of seven cities, Which may lead to severe immunosuppressive effects in chickens. In summary, these findings provide new insights to guide molecular epidemiological surveillance of CIAV in Guangdong province.

\section{Introduction}

Chicken infectious anemia virus (CIAV) is an important causative agent of immunosuppressive disease in chickens worldwide [1]. The first CIAV case was isolated from a vaccine in Japan [2, 3], whereas in China, the first isolated case was reported in 1992, from a local market in the north and east mainland regions [4]. In southern China, research on the live poultry market shows that the virus's prevalence is as high as $87 \%$ [5]. In recent years, poultry surveys have reported that the seropositivity rate of CIAV is high across farms in 3 provinces in China (Zhejiang, Jiangsu, and Anhui) [6]. These data indicate the widespread distribution of CIAV in China.

The CIAV virion is non-enveloped, icosahedral, and approximately about $25 \sim 26.5 \mathrm{~nm}$ [7]. Its genome sequence is highly conserved, containing 3 ORFs (Open Reading Frames), VP1 (1350 bp), VP2 (651 bp), and VP3 (366 bp) [8, 9]. The ORF-encoding proteins with non-structural proteins (VP2, VP3) and capsid protein (VP1) [10-12] are considered primary immunogenicity proteins [10-11].

Data from whole-genome analysis across public databases reveal that CIAV is classified into four clusters, namely: A, B, C, and D [13]. Globally, Clusters A and B are the most common [14]. In China, clusters $A$ and $D$ are reported to be on the rise $[15,16]$. However, little is known regarding the epidemiology of CIAV in Guangdong province, despite the high chicken density. We believe our data have the potential to increased attention and concern in this field.

\section{Methods}

\section{Cells}


The chicken lymphoblastoid cell line MDCC-MSB1 [17] was used for propagation and infectivity titration of CIAV. Cells were cultured in RPMI 1640 medium (Gibco, USA) supplemented with $10 \%$ fetal bovine serum (Gibco, USA) and 1\% penicillin-streptomycin (Hyclone, USA), at $37^{\circ} \mathrm{C}$ in $5 \% \mathrm{CO}_{2}$ atmosphere.

\section{Samples collection}

We collected 325 specimens from 14 breeding farms that did not vaccinate their chickens against CIAV in Guangzhou, Huizhou, Qingyuan, Jiangmen, Zhuhai, Zhongshan, Shantou between July 2018 and March 2019. Besides, four tissue samples were obtained from breeder farms in Huizhou, Jiangmen, and Lufeng. The tissues were washed twice with cold sterile PBS, homogenized, and centrifuged at $12000 \mathrm{rpm}$ for 10 min at $4{ }^{\circ} \mathrm{C}$. The supernatant was inoculated with $1 \%$ Strepto-penicillin (Sigma, USA), and the contents were stored at $-80{ }^{\circ} \mathrm{C}$ for further use.

\section{Cloning and sequencing of the viral genome}

DNA was extracted from the tissue samples using the AxyPrep Body Fluid Viral DNA/RNA Miniprep Kit (Axygen, USA) following the manufacturer's instructions. DNA quality and quantity were determined using a spectrophotometer, and the samples were stored at $-20^{\circ} \mathrm{C}$ until further use.

PCR was used to detect CIAV by targeting the VP1, VP2, and VP3 genes. PCRs were performed using the Premix $\operatorname{Taq}^{\text {TM }}$ (Takara, Japan). Thermocycling conditions were as follows: 4 min denaturation step at $94^{\circ} \mathrm{C}$, followed by 30 cycles of $30 \mathrm{~s}$ at $94^{\circ} \mathrm{C}, 30 \mathrm{~s}$ at $53^{\circ} \mathrm{C}$, and $45 \mathrm{~s}$ at $72^{\circ} \mathrm{C}$ with a 10 min elongation step at $72^{\circ} \mathrm{C}$. Primer sequences are presented in Table 1.

Table 1 Primers used for the identification of CIAV and amplifying Full-length VP1, VP2, and VP3 genes.

\begin{tabular}{|c|c|c|}
\hline Primer name & Sequence $₫ 5^{\prime}$ to $3^{\prime} \rrbracket$ & Length \\
\hline CIAV-F & GCTCTCCAAGAAGATACTCCA & \multirow[t]{2}{*}{$723 \mathrm{bp}$} \\
\hline CIAV-R & TATGTTAGGTTCATTGACGCT & \\
\hline CIAV-F684 & AGTAGGTATACGCAAGGCGGT & \multirow[t]{2}{*}{684 bp } \\
\hline CIAV-R684 & AGCCTCACACTATACGTA & \\
\hline CIAV-F1243 & GCGGTATCGTAGACGAGC & \multirow[t]{2}{*}{$1243 \mathrm{bp}$} \\
\hline CIAV-R1243 & СССTTTTCAGGGCTGCG & \\
\hline
\end{tabular}

\section{Isolation of the virus}

Primers for the VP1 gene were designed for CIAV detection. The virus was isolated from all PCR-positive tissue, $200 \mu \mathrm{L}$ inoculated into the yolk sac cavity and allantoic sac of 9-day-old SPF embryonated chicken eggs, harvested in 19-day-old, and blind passaged three times. 
MSB1 cells $\left(2.5 \times 10^{5}\right.$ cells $\left./ \mathrm{mL}\right)$ in suspension culture supplemented with $10 \%$ FBS and $1 \%$ penicillinstreptomycin were inoculated with the tissue supernatant at a ratio of 1:10. Inoculated cells were subcultured every 2-4 days by adding fresh medium at a rate of $1: 5$. Cells were examined regularly to assess the cytopathic effects (CPE).

\section{Indirect immunofluorescence assays (IFA)}

The CIAV caused little or no damage to MSB1 cells. Consequently, indirect immunofluorescence assays (IFA) were used to estimate the infection rate based on the number of MSB1 cells infected by CAIV. Pathological cells were fixed in $4 \%$ paraformaldehyde (Solarbio, China) for $10 \mathrm{~min}$ and then blocked with $1 \%$ BSA for $30 \mathrm{~min}$. They were subsequently incubated with positive sera (500 fold dilution) for $1 \mathrm{~h}$ and washed thrice with PBS. This was followed by incubation with goat anti-chicken IgY (1000-fold dilution) for $1 \mathrm{~h}$ in the dark. Fluorescing MSB1 cells were observed, counted, and photographed under an inverted fluorescence microscope.

\section{Sequence analysis}

DNAman software was used to analyze sequence data and splice full-length nucleotide sequences of the CIAV genome. We manually edited the sequences using Bioedit (Version 7.12) software and used them as queries to identify and retrieve identical CIAV nucleotide sequences in the GenBank, using the Basic Local Alignment Search Tool (BLAST). Multiple sequence alignments were performed using the ClustalX algorithm, followed by phylogenetic analysis using the Maximum likelihood algorithm in MEGAX (Version 10.1.8), and based on the Tamura-Nei model and 1000 bootstrap replications.

\section{Results}

\section{Identification of CIAV}

Serological analysis identified over $60 \%$ positive cases for CIAV (202/325) and all the cases were recorded across each city (Fig. 1). Across breeder farms, Zhuhai, Qingyuan and Jiangmen, recorded 56.67 (17/30), 70.97 (22/31), and 85.71\% (24/28) positive cases, respectively. Besides, 88.16 (67/76), 100 (19/19), and $100 \%(26 / 26)$ of samples collected from broilers in Huizhou, Zhongshan, and Shantou, respectively, were positive. Notably, we found no positive cases across 16 serum samples collected from single breeds of chicken. In contrast $73.33 \%$ (11/15) of the remaining single chicken breeds were positives for CIAV from the same farm in Foshan. Similarly, 22 samples from breeder farms were negative for CIAV (Fig. 1). Overall, these findings showed that biosafety-based management plays an essential role in the reduction of CIAV transmission.

\section{PCR-based CIAV detection}

Agarose gel electrophoresis showed that all four tissue samples were positive for CIAV (Fig. 2).

\section{Phylogenetic analysis}


Analysis of VP1, VP2, and VP3 genes in the four isolates revealed neither nucleotide insertions nor deletions. The VP1 amino acid hypervariable sequences in the four isolates were KSQAAENWPNCWL. This sequence contributed to host-associated pathogenicity and cell tropism[6]. The four CIAV strains in VP1 share the same glutamine (Q) at position 394, which is an essential determinant of virulence, and previous studies show that it might be highly pathogenic[9]. Nucleotide sequence data reported are available in the GenBank databases under the accession numbers CIAV-GDHZ2-VP1, MW817563; CIAVGDHZ1-VP1, MW817564; CIAV-GDJM-VP1, MW817565; CIAV-GDLF-VP1, MW817566; CIAV-GDHZ1-VP2, MW817567; CIAV-GDHZ2-VP2, MW817568; CIAV-GDJM-VP2, MW817569; CIAV-GDLF-VP2, MW817570; CIAV-GDHZ1-VP3, MW817571; CIAV-GDHZ2-VP3, MW817572; CIAV-GDJM-VP3, MW817573; CIAV-GDLFVP3, MW817574.

There were six single amino acid substitutions across the four CIAV genes (Table 2).

Table 2 Comparison of amino acid sequence mutations in the four CIAV genes

\begin{tabular}{|lllllll|}
\hline Gene & \multicolumn{3}{l}{ VP1 } & & VP2 & VP3 \\
\cline { 2 - 7 } & \multicolumn{3}{l}{ Amino acid position } \\
Strain & 157 & 265 & 281 & 287 & 221 & 115 \\
CIAV-GDLF & M & T & D & S & G & G \\
CIAV-GDJM & V & A & G & N & G & G \\
CIAV-GDHZ1 & V & T & D & S & G & G \\
CIAV-GDHZ2 & V & T & D & S & A & A \\
\hline
\end{tabular}

Nucleotide identities for VP1 ranged between 98.7 99.3\%. Compared with other domestic and overseas epidemic strains, VP1 showed $94.1 \sim 99.6 \%$ and $94.6 \sim 99.0 \%$ identities, respectively. VP2 in the four CIAV strains showed identities ranging between $99.7 \sim 100 \%$, while comparisons with other domestic and overseas epidemic strains resulted in $98.5 \sim 100 \%$ and $98.8 \sim 100 \%$, respectively. Finally, VP3 showed identities ranging between $99.7 \sim 100 \%$ in the four CIAV strains, with $98.6 \sim 100$, and $98.4 \sim 100 \%$ similarities to domestic and overseas strains.

The maximum likelihood algorithm, based on VP1 of the CIAV strains in this study, yielded lower scores; hence we adopted the neighbor-joining method for constructing a phylogenetic tree (Fig. 3). Most of the overseas strains were clustered in clades A and B. Those from Australia formed a single clade, while most domestic strains from China, including the four strains in this study, were found in clade C.

CIAV-YM strain (Vaccine Strain), together with CIAV-F10 and CIAV-N22, clustered into clade B. Therefore, the four strains isolated in this study did not mutate from the vaccine strains because of the long genetic distance. The four strains were located within the same branch C2. But CIAV-GDHZ2 was closely related to CIAV-GD-N-12 and CIAV-GD-L-12, isolated from Guangdong province (clade C2). Notably, CIAV-GDHZ1 
and CIAV-GDHZ2 strains were both isolated from the same chicken breed on the same farm.

Nevertheless, the phylogenetic tree showed that they did not have a close distance in relatedness, suggesting that there may have been more than one strain on the same farms at the same time (Fig. 3).

\section{CIAV isolation from MSB1 cells}

Primers for the VP1 gene were used to detect CIAV. PCR-positive tissue homogenate supernatant was inoculated into 9-day-old SPF embryonated chicken eggs and blind-passaged three times. However, none of the eggs tested positive for the virus.

Inoculation of MSB1 cells with the same tissue homogenate supernatant induced cytopathic changes, such as cell swelling, fragmentation, and increased debris, after continuous passage as revealed by microscopic examination. Serial passage of the four CIAV isolates onto MSB1 cells for 5 to 6 generations showed marked cytopathies under the microscope (Fig. 4). These cytopathies were consistent with those reported by Cardona[18].

\section{Indirect immunofluorescence assays (IFA)}

After MSB1 cells were infected with CIAV-GDJM for $72 \mathrm{~h}$ and blind passaged six times, they were subjected to indirect immunofluorescence assay. Results showed green fluorescence in the test group (Fig. $5 \mathrm{~A}$ and $\mathrm{B}$ ) but not in the control group (Fig. $5 \mathrm{C}$ and D). These findings confirmed that the virus isolated in this experiment was CIAV.

\section{Discussion}

Chicken farming and consumption is high in China, with Guangdong being the critical region for industrial poultry breeding and consumption. Effective control of poultry diseases is an essential goal for poultry breeding in the country. Several studies have investigated CIAV, which causes high mortality rates and induces immunosuppression. Recently, high positive CIAV antibody rates in chickens have attracted considerable interest in China. Our results revealed that chickens in parts of Jiangsu had a positive rate of CIAV antibody of $39.1 \%$, whereas those in Anhui had a $63.6 \%$ rate. However, the epidemiological characteristics of CIAV in Guangdong province are not well-known.

\section{Serological analysis of CIAV strains}

Analysis of 325 serum samples in this study revealed visible clinical symptoms in most chicken, and a $62.15 \%$ positive rate of CIAV antibody was reported. These results suggest the occurrence of CIAV in more regions. Our results showed that two different chicken breeds had completely different CIAV positive rates on the same farm, indicating that the response to CIAV may be breed-specific. Moreover, we observed a considerable difference in the positive rate of CIAV between the breeder farms and six free-range chicken households across Huizhou. The observed increase in positive CIAV rates may be attributed to the higher stocking density of all the households relative to reasonable stocking rates in the breeder farm. A strict bio-safety management approach can reduce CIAV cases. We detected positive CIAV cases in 6 of the 
seven breeding farms. Since CIAV can be transmitted vertically from the female to the offspring, the actual number of CIAV infections may be higher than observed herein. Although the total number of samples collected in this study was limited, we selected sampling sites according to the breeding density of chickens in Guangdong province. The seven chosen cities herein yielded samples that were positive for CIAV. Therefore, significant attention should be directed to CIAV infections in Guangdong province. To effectively prevent and control CIAV, strict bio-safety management measures must be combined with quarantine in all farms and households to prevent transmission.

\section{Phylogenetic analysis of CIAV strains}

Previous studies have shown that the amino acid glutamine (Q) at position 394 in VP1 determines CIAV's pathogenicity [7]. The virus with glutamine was highly pathogen and low pathogenicity for viruses with histidine [19]. Analysis of the CIAV strains across the Genbank database revealed that most of the residue 394 of the VP1 protein was also glutamine. In this study, all CIAV strains isolated from chickens had similar sites represented by high pathogenicity [20]. Previous studies report that amino acids at positions 139 and 144 were not glutamine, indicating that the viruses may promote viral growth and spread. Our sequence analysis showed that the VP1 proteins in this study were highly homologous $(94.1 \% \sim 99.6 \%)$ relative to others across the database, as well as VP2 and VP3 proteins. A comparison among all three proteins revealed relatively less conservation[21], indicating that VP1 protein is the only capsid protein exposed on the virion surface. This may be due to selection pressure and the immune response. There are four mutations outside the hypervariable region of VP1, V157M, T265A, D281G, and S287N, which have never been previously described. Future research should pay attention to the effects of the four mutations in pathogenicity, replication, and host range on CIAV. Moreover, all sequences were relatively conserved, and epidemiological analysis showed that CIAV strains in Guangdong province mainly clustered in clade $\mathrm{C} 2$, with all $4 \mathrm{CIAV}$ isolates located in this clade. Besides, a significant genetic distance was noted between the four isolates and CIAV-YM strains (Vaccine Strain) (Fig. 3). CIAV-GDHZ1 and CIAV-GDHZ2 strains from the same batches on the same farm recorded a relatively large genetic distance with the other two CIAV in this study. Our study reveals possible multiple CIAV infections on the same farm.

\section{Isolation of CIAV strains}

Viral isolation by using specific pathogen-free (SPF) embryonated chicken eggs or MDCC-MSB1 cells, can provides a favorable proliferation environment [17]. In the present study, we used both methods (SPF embryonated chicken eggs and MSB1 cells) to isolate the virus. The results suggested that different isolation methods affect CIAV proliferation differently. Follow-up analysis using PCR revealed that the samples were negative for CIAV and that the virus did not proliferate under these conditions (Inoculation of SPF embryonated chicken eggs). The reason for this negative effect is obvious, and several factors may be involved, such as human factors. Nevertheless, these results were attributed to several amino acid mutations. Besides, how the virus-host interactions influence the outcome remains an important question. Microscopic analysis following virus isolation revealed morphological changes, including cellular swelling, fragmentation, and other cytopathic changes. Indirect immunofluorescence microscopy 
assay also showed green fluorescence in the test group. These data prove that CIAV spread during this study differed significantly in hosts. Factors that influence virus-host interaction require further investigation.

\section{Conclusion}

In conclusion, we included 325 serum samples and 4 tissue samples collected from breeding farms in seven major cities of Guangdong province between July 2018 and March 2019 to characterize and isolate CIAV strains. The overall CIAV positivity rate was close to $63 \%$.

All four tissue samples CIAV genome share a common characteristic (nucleotide homology 98.7\% 99.3\%). Based on the phylogenetic analysis, Clade C2 appears more strongly associated with the four virus isolates, and four samples were collected from the same location but divided into two groups. Those data indicate that the CIAV might have mixed infections with several poultry diseases, and the mechanism of CIAV induced immunosuppression is still unclear. This study may give the information generated could be used for epidemiological surveillance and preventive measures.

\section{Declarations}

Acknowledgements The authors wish to thank the veterinary practitioners for their help with sample collection and lab members for their assistance with internal review of the paper.

Funding This study was supported by Natural Science Foundation of Guangdong Province, China (Grant No.2020A1515010116); Medical Scientific Research Foundation of Guangdong Province, China (Grant No. A2020083); Modern agricultural industry technology system innovation team of Guangdong Province (Grant No.2019KJ137); National Key Research and Development Project (Grant No. 2017YFD0500800).

Competing interests The authors declare that there is no conflict of interests regarding the publication of this article.

Availability of data and materials The datasets used and/or analysed during the current study are available from the corresponding author on reasonable request.

Authors' contributions FC Z, WF L and KM performed experiments, analyzed the data and drafted the manuscript; JS H contributed to experimental design, supervised the study, and revised the manuscript. All authors read, commented on and approved the final version of the manuscript.

Ethics approval and consent to participate This study was approved by the Research Ethics Committee of the College of Life Science and Engineering, Foshan University.

Consent for publication not applicable

\section{References}


1. Sun F, Pan W, Gao H, Qi X, Qin L, Wang Y, Gao Y, Wang X (2018) Identification of the interaction and interaction domains of chicken anemia virus VP2 and VP3 proteins. Virology 513:188-194. https://doi.org/10.1016/j.virol.2017.09.014

2. Yuasa N, Taniguchi T, Yoshida I: Isolation and some characteristics of an agent inducing anemia in chicks. Avian diseases 1979:366-385

3. Yuasa N: Propagation and infectivity titration of the Gifu-1 strain of chicken anemia agent in a cell line (MDCC-MSB1) derived from Marek's disease lymphoma. National Institute of Animal Health Quarterly, Japan 1983, 23(1):pp 13-20

4. Cui XL, Xing GX, Wu DL, Feng JY, Li X, Qu LX: Identification of chicken infectious anaemia virus. Chinese Journal of Preventive Veterinary Medicine, China 1992(6):3-5

5. Ducatez MF, Chen H, Guan Y, Muller CP (2008) Molecular epidemiology of chicken anemia virus (CAV) in southeastern Chinese live birds markets. Avian Dis 52(1):68-73. https://doi.org/10.1637/8049-070407-Reg

6. Sun BB, Zhang CL, Gui PX (2019) The Research Progress of Chicken infectious Anemia. Zhejiang Journal Animal Science Veterinary Medicine China 44(02):8-10

7. van Santen VL, Li L, Hoerr FJ, Lauerman LH (2001) Genetic characterization of chicken anemia virus from commercial broiler chickens in Alabama. Avian Dis 45(2):373-388

8. Renshaw RW, Soiné C, Weinkle T, O'Connell PH, Ohashi K, Watson S, Lucio B, Harrington S, Schat KA (1996) A hypervariable region in VP1 of chicken infectious anemia virus mediates rate of spread and cell tropism in tissue culture. Journal of virology 70(12):8872-8878. https://doi.org/10.1128/JVI.70.12.8872-8878.1996

9. Islam M, Johne R, Raue R, Todd D, Müller H (2002) Sequence analysis of the full-length cloned DNA of a chicken anaemia virus (CAV) strain from Bangladesh: Evidence for genetic grouping of CAV strains based on the deduced VP1 amino acid sequences. Journal of Veterinary Medicine Series B 49(7):332-337. https://doi.org/10.1046/j.1439-0450.2002.00581.x

10. Noteborn M, Verschueren C, Koch G, Van der Eb AJ (1998) Simultaneous expression of recombinant baculovirus-encoded chicken anaemia virus (CAV) proteins VP1 and VP2 is required for formation of the CAV-specific neutralizing epitope. J Gen Virol 79(12):3073-3077. https://doi.org/10.1099/00221317-79-12-3073

11. Douglas A, Phenix K, Mawhinney K, Todd D, Mackie D, Curran W (1995) Identification of a 24 kDa protein expressed by chicken anaemia virus. Journal of general virology 76(7):1557-1562. https://doi.org/10.1099/0022-1317-76-7-1557

12. Wang Y, Song X, Gao H, Wang X, Hu Y, Gao Y, Qi X, Qin L, Lin H, Gao L (2017) C-terminal region of apoptin affects chicken anemia virus replication and virulence. Virol $\mathrm{J}$ 14(1):1-10. https://doi.org/10.1186/s12985-017-0713-9

13. Erfan AM, Selim AA, Naguib MM (2018) Characterization of full genome sequences of chicken anemia viruses circulating in Egypt reveals distinct genetic diversity and evidence of recombination. Virus Res 251:78-85. https://doi.org/10.1016/j.virusres.2018.05.008 
14. Gowthaman V, Singh SD, Dhama K, Barathidasan R, Srinivasan P, Mahajan NK, Ramakrishnan MA (2014) Molecular characterization of chicken infectious anemia virus isolated from commercial poultry with respiratory disease complex in India. Adv Anim Vet Sci 2(3):171-176

15. Eltahir YM, Qian K, Jin W, Wang P, Qin A (2011) Molecular epidemiology of chicken anemia virus in commercial farms in China. Virol J 8(1):145. https://doi.org/10.1186/1743-422X-8-145

16. Kim H-R, Kwon Y-K, Bae Y-C, Oem J-K, Lee O-S (2010) Molecular characterization of chicken infectious anemia viruses detected from breeder and broiler chickens in South Korea. Poult Sci 89(11):2426-2431. https://doi.org/10.3382/ps.2010-00911

17. Akiyama Y, Kato S (1974) Two cell lines from lymphomas of Marek's disease. Biken J 17(3):105116

18. Cardona CJ, Oswald WB, Schat KA (2000) Distribution of chicken anaemia virus in the reproductive tissues of specific-pathogen-free chickens. J Gen Virol 81(Pt 8):2067-2075. https://doi.org/10.1099/0022-1317-81-8-2067

19. Yamaguchi S, Imada T, Kaji N, Mase M, Tsukamoto K, Tanimura N, Yuasa N (2001) Identification of a genetic determinant of pathogenicity in chicken anaemia virus. J Gen Virol 82(Pt 5):1233-1238. https://doi.org/10.1099/0022-1317-82-5-1233

20. Li Y, Hu Y, Cui S, Fu J, Wang Y, Cui Z, Fang L, Chang S, Zhao P (2017) Molecular characterization of chicken infectious anemia virus from contaminated live-virus vaccines. Poult Sci 96(5):1045-1051. https://doi.org/10.3382/ps/pew406

21. Pallister J, Fahey KJ, Sheppard M (1994) Cloning and sequencing of the chicken anaemia virus (CAV) ORF-3 gene, and the development of an ELISA for the detection of serum antibody to CAV. Vet Microbiol 39(1-2):167-178. https://doi.org/10.1016/0378-1135(94)90097-3

\section{Figures}




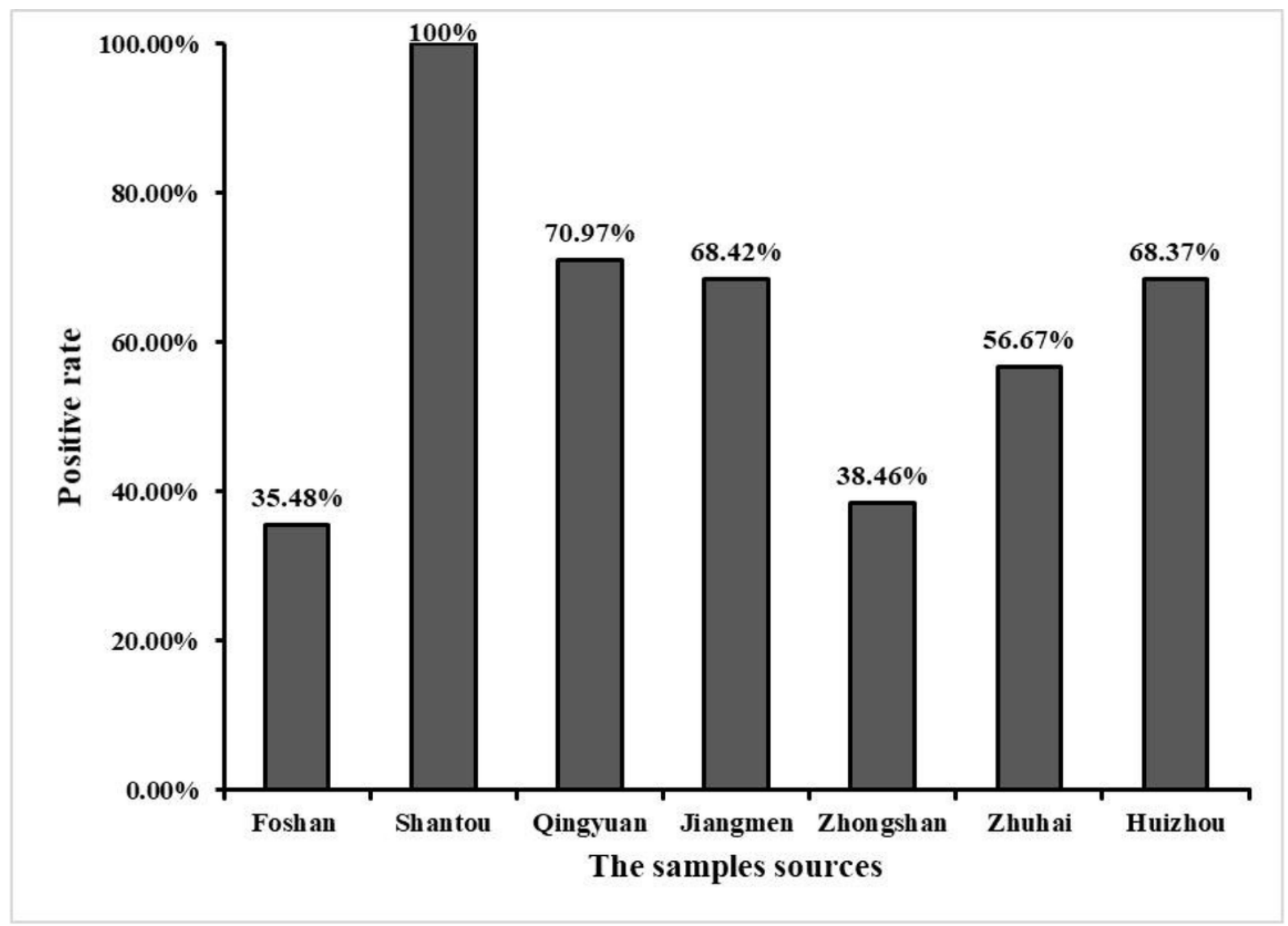

Figure 1

Profiles of positive CIAV cases across each sampled area (Positive nucleic acid detection). 


\section{M \\ 12 \\ 3 \\ 4 \\ 5 \\ 6}

(bp)
2000

1000

750

500

250

100

\section{Figure 2}

Detection of VP1 gene from CIAV in sampled tissues using PCR. M: A 2000 bp DNA marker 1: negative control, 2: Positive control, 3: CIAV-GDJM (723 bp), 4: CIAV-GDLF (723 bp), 5: CIAV-GDHZ1 (723 bp), 6: CIAV-GDHZ2 (723 bp). 


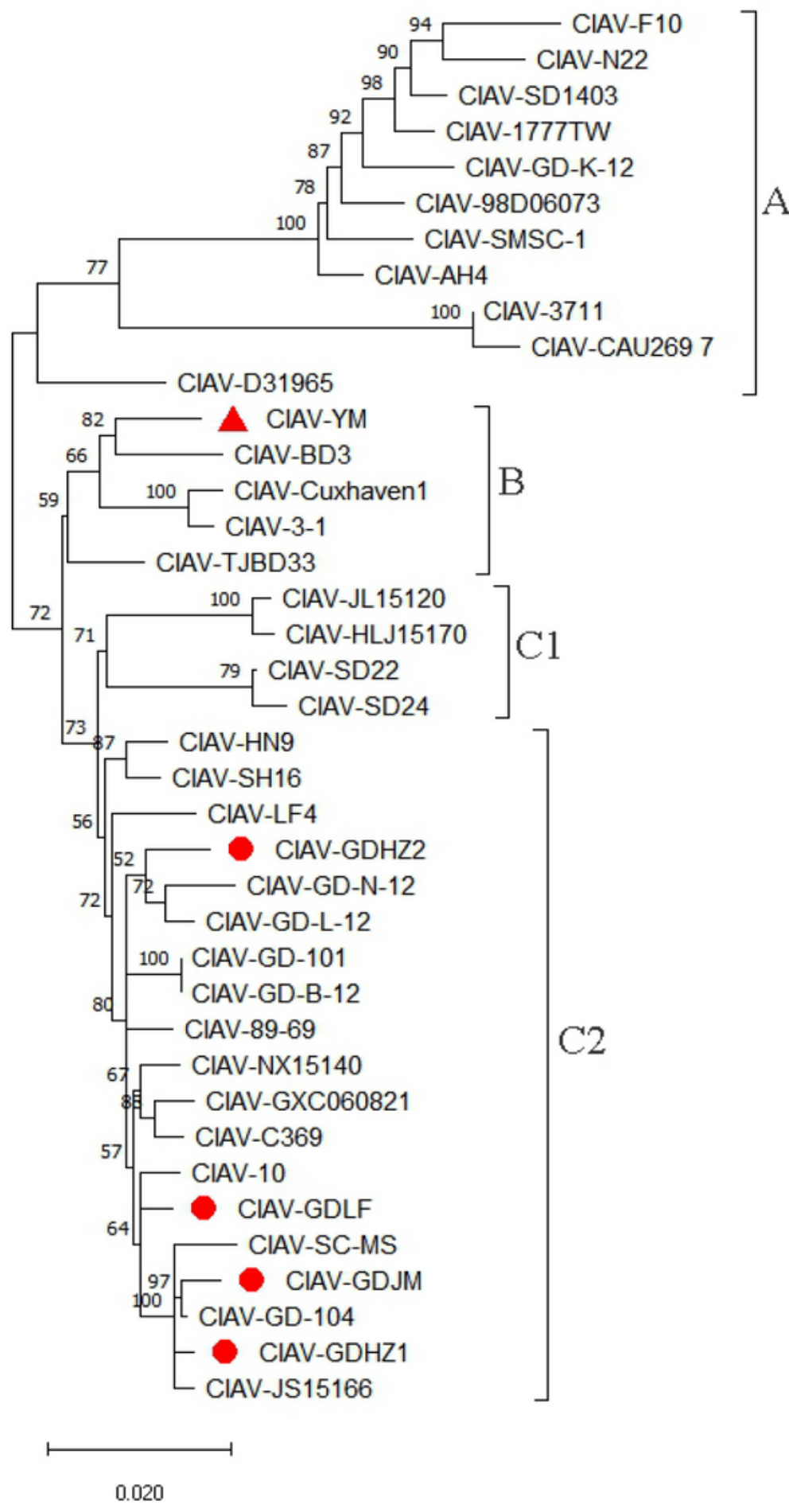

\section{Figure 3}

Phylogenetic analysis of the nucleic acid sequence of VP1 protein using the Maximum likelihood algorithm implemented in MEGAX (Version 10.1.8). Circles represent the four CIAV strains isolated, and triangles represent the vaccine strains. 

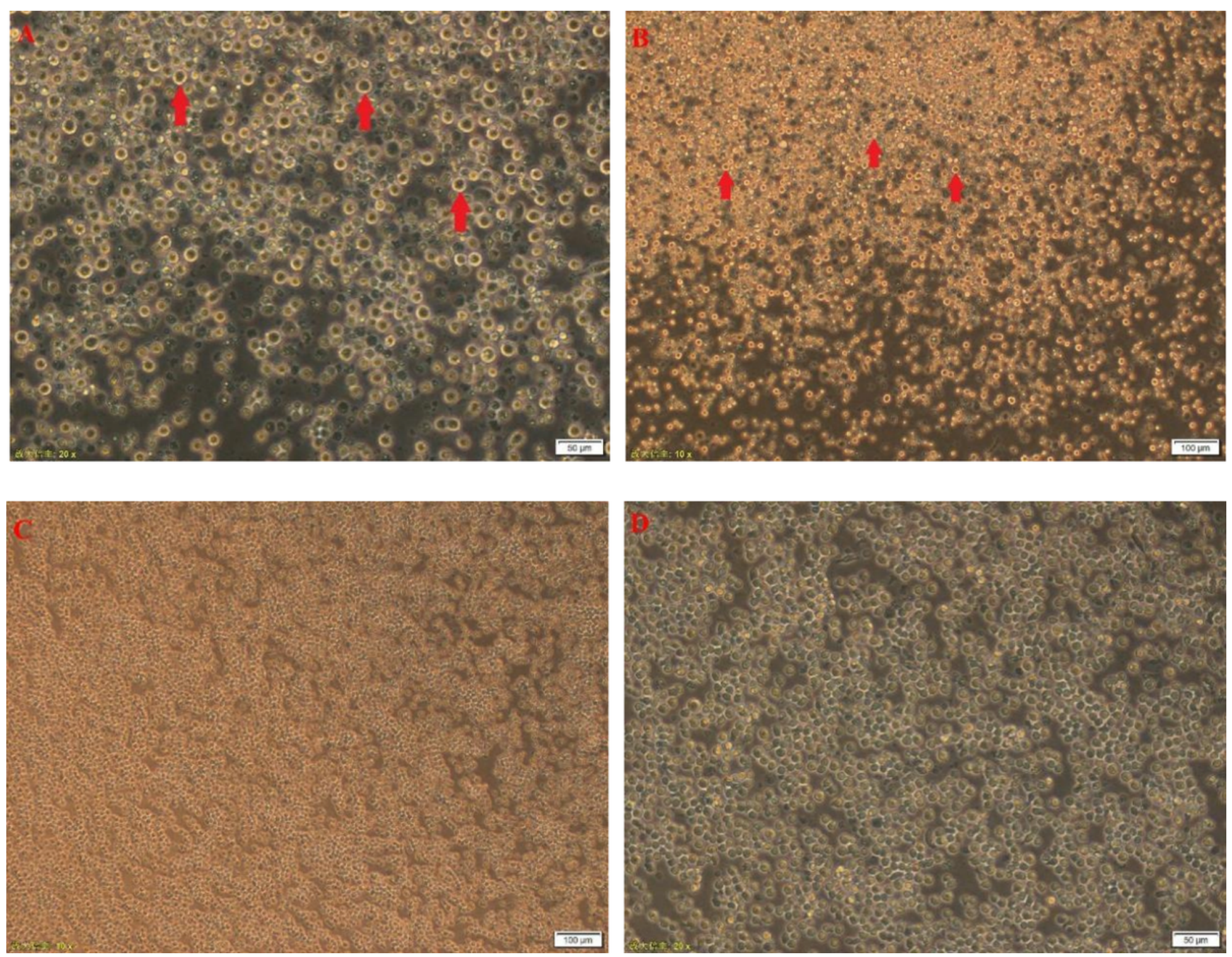

Figure 4

CIAV-GDJM uploading to the 6th generation CPE in MSB1. A囚Experimental group (200x)『B囚Experimental

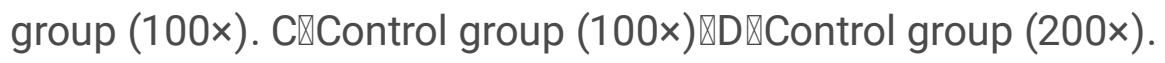




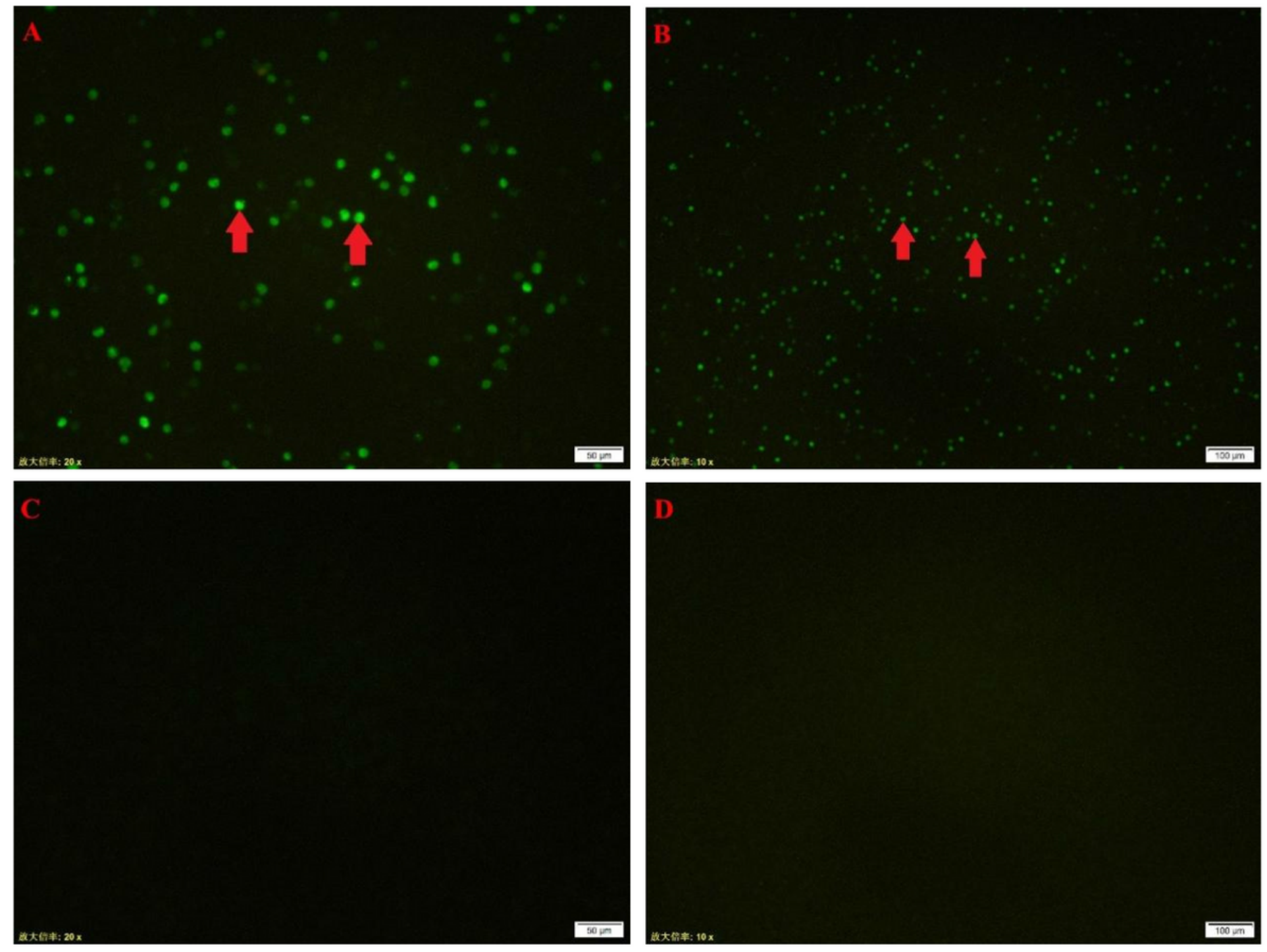

\section{Figure 5}

IFA infected with MSAV1 cells by CIAV-GDJM for $72 \mathrm{~h}$. A: Experimental group within $72 \mathrm{~h}$ post-infection (200x). B: Experimental group within $72 \mathrm{~h}$ post-infection (100x). C: Control group without infection after $72 \mathrm{~h}(200 x)$. D: Control group without infection after $72 \mathrm{~h}(100 \times)$. 